DTP/94-39

October 1994

\title{
Integrable boundary conditions for classical sine-Gordon theory
}

\author{
A. MacIntyre \\ Department of Mathematical Sciences, \\ University of Durham, Durham DH1 3LE, U.K.
}

\begin{abstract}
The possible boundary conditions consistent with the integrability of the classical sine-Gordon equation are studied. A boundary value problem on the half-line $x \leq 0$ with local boundary condition at the origin is considered. The most general form of this boundary condition is found such that the problem be integrable. For the resulting system an infinite number of involutive integrals of motion exist. These integrals are calculated and one is identified as the Hamiltonian. The results found agree with some recent work of Ghoshal and Zamolodchikov.
\end{abstract}

\section{Introduction}

The problem of factorizable scattering on a half-line necessarily involves the so-called "reflection equation" [1, 2]. This equation supplements the Yang-Baxter relation for the factorization of three particle scattering and represents the factorization of two particle scattering in the presence of a one-dimensional 'wall' at $x=0$. Recently there has been increased interest in this problem, and solutions have been suggested for sine-Gordon, affine Toda, and $O(N)$ sigma models 3, 4, 5, 6.

Equations of "reflection" type are also used within the framework of the quantum inverse scattering method [7] to prove the integrability of open quantum spin chains, i.e. chains without periodic boundary conditions 8, 9, 10]. The classical limit of these results was developed in a paper by Sklyanin [11], where it was found that they generalize the Hamiltonian, ( $r$-matrix), approach to inverse scattering theory [12], to the case of nonlinear partial differential equations with local boundary conditions. The adaptation of these results to inverse scattering theory on the half line $x \leq 0$ with a local boundary condition at $x=0$ [13, 14 saw the problem continued evenly to the whole line with a 'point-spin' impurity at the orgin. These results are equivalent to the approach in [15, 16] where symmetrical reductions of Bäcklund transformations were considered. The analysis of these problems on a finite interval is to be found in 17, 18 .

Ghoshal and Zamolodchikov [3], conjectured both the classical and quantum integrability of the sineGordon theory on the half line with a boundary term at the origin. A classical action is,

$$
S=\int_{-\infty}^{\infty} d t \int_{-\infty}^{\infty} d x\left[\Theta(-x)\left(\frac{1}{2}\left(\partial_{\mu} \phi(x, t)\right)^{2}-\frac{m^{2}}{\beta^{2}}(1-\cos \beta \phi(x, t))\right)+\delta(x) V(\phi(x, t))\right],
$$

the minimisation of which leads to the boundary value problem,

$$
\begin{aligned}
\partial^{\mu} \partial_{\mu} \phi(x, t)+\frac{m^{2}}{\beta} \sin \beta \phi(x, t) & =0 \quad x \leq 0, \\
\frac{\partial \phi(x, t)}{\partial x}+\frac{\partial V(\phi(x, t))}{\partial \phi} & =0 \quad x=0, \\
\lim _{x \rightarrow-\infty}(\phi(x, t)(\bmod 2 \pi / \beta)) & =0,
\end{aligned}
$$

with the boundary value at $-\infty$ being attained in the sense of Schwartz, i.e. $\phi=0(\bmod 2 \pi / \beta)$ and all its derivatives decay faster than any inverse power of $x$ as $x \rightarrow-\infty$. 
By demanding that a modification of the first non-trivial integral of motion of the bulk theory remain conserved on including the boundary it was found [3] that in general,

$$
V(\phi(x, t))=\frac{A m}{\beta} \cos \left(\frac{\beta\left(\phi(x, t)-\phi_{0}\right)}{2}\right),
$$

where $A$ and $\phi_{0}$ are arbitrary real constants. In [3] the existence of an infinite number of integrals of motion for the system (2),(3) was assumed and the associated quantum field theory was studied.

In this paper, the question of integrability of the classical system (2) is addressed. The results of Sklyanin are used to prove that an infinite number of integrals of motion do exist, but only for certain $V(\phi)$. It is found that (3) is the most general boundary term compatible with the integrability of (2). The results thus agree with Ghoshal and Zamolodchikov. Further analysis of the system (2),(3) can be found in [19].

In section 2 the auxiliary linear problem of inverse scattering theory is explained. The case of periodic boundary conditions is considered first, followed by the calculation of the integrals of motion for sine-Gordon. Next follows Sklyanin's extension to other types of boundary value problem.

Finally, in section 3 the formalism developed is applied to the system (2), and the requirement on the form of the boundary term $V(\phi)$ is deduced. The integrals of the resulting boundary value problem are studied and the Hamiltonian is identified.

The notation of 12 is used throughout the following sections.

\section{The auxiliary linear problem of inverse scattering theory}

\subsection{Periodic boundary conditions}

The zero-curvature approach to inverse scattering [12] relies on the existence of a pair of linear partial differential equations for a $d \times d$ matrix whose elements are maps from $\mathbb{R}^{2} \times \mathbb{C}$ to $\mathbb{C}$. The solution of such a system subject to a certain initial condition defines the transition matrix $T(x, y, t, \alpha)$ uniquely. Suppressing the argument of $T(x, y, t, \alpha)$, and understanding that $x \geq y$ throughout, the pair of equations and the initial condition are,

$$
\begin{aligned}
\frac{\partial T}{\partial x} & =U(x, t, \alpha) T \\
\frac{\partial T}{\partial t} & =V(x, t, \alpha) T-T V(y, t, \alpha) \\
T(y, y, t, \alpha) & =I .
\end{aligned}
$$

Here, $U(x, t, \alpha), V(x, t, \alpha)$ are $d \times d$ matrices whose elements are functions of a complex valued field $\Phi(x, t)$, its derivatives, and a spectral parameter $\alpha \in \mathbb{C}$.

The formal solution to (4), (6) is given by,

$$
T(x, y, t, \alpha)=\mathcal{P} \exp \left\{\int_{y}^{x} U\left(x^{\prime}, t, \alpha\right) d x^{\prime}\right\},
$$

where $\mathcal{P}$ denotes path ordering of noncommuting factors, 12 .

Compatibility of (4) and (5) gives the zero curvature condition,

$$
\frac{\partial U}{\partial t}-\frac{\partial V}{\partial x}+[U, V]=0, \quad \forall \alpha \in \mathbb{C}
$$

and this should imply the desired nonlinear evolution equation for $\Phi$.

For the sine-Gordon equation, $U$ and $V$ are $2 \times 2$ matrices and can be written in terms of the standard Pauli matrices $\sigma_{k}, k=1,2,3$ and a real valued field $\phi$ as,

$$
\begin{aligned}
U(x, t, \alpha) & =\frac{\beta}{4 i} \frac{\partial \phi}{\partial t} \sigma_{3}+\frac{m}{2 i} \cosh \alpha \sin \left(\frac{\beta \phi}{2}\right) \sigma_{1}+\frac{m}{2 i} \sinh \alpha \cos \left(\frac{\beta \phi}{2}\right) \sigma_{2} \\
V(x, t, \alpha) & =\frac{\beta}{4 i} \frac{\partial \phi}{\partial x} \sigma_{3}+\frac{m}{2 i} \sinh \alpha \sin \left(\frac{\beta \phi}{2}\right) \sigma_{1}+\frac{m}{2 i} \cosh \alpha \cos \left(\frac{\beta \phi}{2}\right) \sigma_{2} .
\end{aligned}
$$


The imposition of periodic boundary conditions on $\phi(x, t)$ with period $2 L$ ensures periodicity of $V(x, t, \alpha)$ also, and (5) becomes,

$$
\frac{\partial T(L,-L, t, \alpha)}{\partial t}=[V(L, t, \alpha), T(L,-L, t, \alpha)] .
$$

Taking the trace of (11) and using the chain rule for differentiation gives,

$$
\frac{\partial \ln (\operatorname{tr} T(L,-L, t, \alpha))}{\partial t}=0, \quad \forall \alpha,
$$

which can be expanded as a Laurent series in $\alpha$ to give an infinite number of integrals of motion, related to the field $\phi$ by (7). The existence of such an infinite set is crucial for the integrability of the original evolution equation, in particular for the construction of action-angle variables for the theory.

In the limit $L \rightarrow \infty$, eq.(11) simplifies as $\phi$ and its derivatives vanish, and the evolution of the matrix elements of $T$, the transition coefficients, have a simple form. A detailed treatment of the auxiliary linear problem (4), and its inversion, the Riemann problem, can be found in [12].

The alternative formulation to the one outlined above is the so-called Hamiltonian, or $r$ matrix, approach. The problem is now regarded as an infinite dimensional Hamiltonian system with phase space $\mathcal{M}$. Points in this space are determined by pairs of complex $2 L$ spatially periodic functions at fixed time $(\Phi(x, t), \Pi(x, t))$, $\Pi$ being the conjugate momentum to $\Phi$. The 'algebra of observables' $\mathcal{A}$ on $\mathcal{M}$ is defined over $\mathbb{C}$ and comprises functionals $A: \mathcal{M} \rightarrow \mathbb{C}$, bounded everywhere and having smooth functional derivatives that are $2 L$ periodic functions of $x$. The Poisson bracket on this algebra is defined at constant time slices by,

$$
\{A, B\}:=\int_{-L}^{L}\left(\frac{\delta A[\Phi(u, t), \Pi(u, t)]}{\delta \Pi(z, t)} \frac{\delta B[\Phi(v, t), \Pi(v, t)]}{\delta \Phi(z, t)}-\frac{\delta A[\Phi(u, t), \Pi(u, t)]}{\delta \Phi(z, t)} \frac{\delta B[\Phi(v, t), \Pi(v, t)]}{\delta \Pi(z, t)}\right) d z,
$$

where $A, B \in \mathcal{A}$ and $u, v, z$ now lie inside the fundamental domain $[-L, L]$. This Poisson bracket can be generalised to act on $d \times d$ matrices, $C, D$ whose elements are in $\mathcal{A}$, by replacing $A, B$ in (13) by $C^{(1)}, D^{(2)}$, respectively, where $C^{(1)}:=C \otimes I, D^{(2)}:=I \otimes D$, and $I$ is the $d \times d$ identity matrix. As a result of this notation, $\left\{C^{(1)}, D^{(2)}\right\}$ is a $d^{2} \times d^{2}$ matrix whose elements are the Poisson brackets of all possible combinations of matrix elements of $C, D$.

The Hamiltonian formulation of the problem relies on the existence of an $r$ matrix. This is a function from $\mathbb{C}$ to End $\left(\mathbb{C}^{d} \otimes \mathbb{C}^{d}\right)$. It is defined in terms of the transition matrix, $T(x, y, t, \alpha)$ (7) by the equation,

$$
\left\{T^{(1)}(x, y, t, \alpha), T^{(2)}(x, y, t, \gamma)\right\}=\left[r_{12}(\alpha-\gamma), T^{(1)}(x, y, t, \alpha) T^{(2)}(x, y, t, \gamma)\right], \quad \forall \alpha, \gamma \in \mathbb{C} .
$$

The dependence of $r$ on the difference $(\alpha-\gamma)$ is not the case for all models, but it is so for the sine-Gordon equation considered below. Antisymmetry and the Jacobi identity for the bracket hold if and only if,

$$
\begin{aligned}
r_{12}(\alpha-\gamma)+r_{21}(\gamma-\alpha) & =0 \\
{\left[r_{12}(\alpha-\gamma), r_{13}(\alpha)+r_{23}(\gamma)\right]+\left[r_{13}(\alpha), r_{23}(\gamma)\right] } & =0
\end{aligned}
$$

where the subscripts denote the nontrivial action of $r$ in $\mathbb{C}^{d} \otimes \mathbb{C}^{d} \otimes \mathbb{C}^{d}$. Eq(16) is the classical Yang-Baxter equation.

For sine-Gordon, phase space $\mathcal{M}_{S G}$ is the subset of $\mathcal{M}$ given by pairs of real $2 L$ periodic functions $(\phi(x, t), \pi(x, t))$

$$
\phi(x+2 L, t) \equiv \phi(x, t) \bmod \frac{2 \pi}{\beta}, \quad \pi(x+2 L, t)=\pi(x, t), \quad \pi(x, t):=\frac{\partial \phi(x, t)}{\partial t} .
$$

On this space will be defined the algebras $\mathcal{A}_{S G}$ and $\mathcal{A}_{S G}^{\mathbb{R}}$. The algebra $\mathcal{A}_{S G}$ is the restriction of $\mathcal{A}$ to $\mathcal{M}_{S G}$, while $\mathcal{A}_{S G}^{\mathbb{R}}$ is the subset of $\mathcal{A}_{S G}$ mapping $\mathcal{M}_{S G} \rightarrow \mathbb{R}$, which becomes an algebra on restricting the scalars of $\mathcal{A}_{S G}$ to $\mathbb{R}$. For convenience, the Poisson bracket will be defined on both these algebras but it is understood that the integrals of motion should be elements of $\mathcal{A}_{S G}^{\mathbb{R}}$. Also, $d=2$ and,

$$
r_{S G}(\alpha-\gamma)=\frac{\beta^{2}}{16 \tanh (\alpha-\gamma)}\left(I \otimes I-\sigma_{3} \otimes \sigma_{3}\right)-\frac{\beta^{2}}{16 \sinh (\alpha-\gamma)}\left(\sigma_{1} \otimes \sigma_{1}+\sigma_{2} \otimes \sigma_{2}\right) .
$$


Returning to the integrals of motion of a general theory, taking the trace of (14) and using the chain rule for functional differentiation gives,

$$
\{\ln (\operatorname{tr} T(x, y, t, \alpha)), \ln (\operatorname{tr} T(x, y, t, \gamma))\}=0, \quad \forall \alpha, \gamma \in \mathbb{C}, \quad x, y \text { s.t. }-L \leq y \leq x \leq L
$$

which, on expansion as Laurent series in $\alpha, \gamma$ about the singularities in $T$, gives an infinite number of involutive charges, depending on $\Phi$ through (7). However, so far no mention has been made of the boundary conditions on $\Phi$. These have been used in defining the 'algebra of observables' $\mathcal{A}$, and the fundamental domain

$-L \leq y \leq x \leq L$. For these involutive quantities to have smooth functional derivatives and so be elements of this algebra requires that $x=L, y=-L$. Hence, the integrals of motion found using the zero-curvature formulation are also in involution with respect to the Poisson bracket (13). This suggests that the model may be completely integrable, along the lines of the Liouville-Arnold theorem [20] for finite dimensional Hamiltonian systems.

Alternatively, there is no need to appeal to the zero-curvature condition at all. By requiring the involutive charges to be elements of $\mathcal{A}$, they are fixed uniquely. Among these should exist a Hamiltonian $H(L,-L, t)$ such that,

$$
\frac{\partial \Phi(x, t)}{\partial t}=\{H(L,-L, t), \Phi(x, t)\}, \quad \frac{\partial \Pi(x, t)}{\partial t}=\{H(L,-L, t), \Pi(x, t)\}, \quad-L \leq x \leq L,
$$

imply the field equation. The problem can then be extended to the $x$ axis by letting $L \rightarrow \infty$ and demanding that $\Phi$ be a Schwartz function.

For the sine-Gordon equation such a Hamiltonian is found as follows. Fixing $\operatorname{Im} \alpha=\pi / 2$, (9) shows $T(L,-L, t, \alpha)$ to have essential singularities at $\operatorname{Re} \alpha= \pm \infty$. As a result, it is expected that there will exist two distinct infinities of involutive integrals coming from the coefficients of the Laurent expansions about these two "points". First consider $\operatorname{Re} \alpha \rightarrow+\infty$. Suppressing $t$ and defining $T^{\prime}(L,-L, \lambda)=T(L,-L, \alpha)$ and $\lambda:=e^{\alpha}$, $T^{\prime}(x, y, \lambda)$ is rewritten as,

$$
T^{\prime}(x, y, \lambda)=\Omega(x) \tilde{T}(x, y, \lambda) \Omega^{-1}(y),
$$

where $\Omega(x)=\exp \left(i \beta \phi(x) \sigma_{3} / 4\right)$. It can be shown [12 that for $\lambda \rightarrow \pm i \infty$,

$$
\tilde{T}(x, y, \lambda)=(I+W(x, \lambda)) \exp Z(x, y, \lambda)(I+W(y, \lambda))^{-1}+O\left(|\lambda|^{-\infty}\right),
$$

where,

$$
\begin{aligned}
W(x, \lambda) & =\sum_{n=0}^{\infty} \frac{W_{n}(x)}{\lambda^{n}} \\
Z(x, y, \lambda) & =\frac{m \lambda(x-y)}{4 i} \sigma_{3}+i \sum_{n=1}^{\infty} \frac{Z_{n}(x, y)}{\lambda^{n}}
\end{aligned}
$$

The $W_{n}, Z_{n}$ have the form,

$$
W_{n}(x)=\left(\begin{array}{cc}
0 & -\bar{w}_{n}(x) \\
w_{n}(x) & 0
\end{array}\right), Z_{n}(x, y)=\left(\begin{array}{cc}
z_{n}(x, y) & 0 \\
0 & -\bar{z}_{n}(x, y)
\end{array}\right)
$$

with,

$$
\begin{gathered}
w_{0}(x)=i \\
w_{n+1}(x)=\frac{2}{i m} \frac{\partial w_{n}(x)}{\partial x}-\frac{\beta}{m}\left(\pi(x)+\frac{\partial \phi(x)}{\partial x}\right) w_{n}(x)+\frac{i}{2} \sum_{k=1}^{n} w_{k}(x) w_{n+1-k}(x) \\
-\frac{i}{2} e^{-i \beta \phi(x)} \sum_{k=0}^{n-1} w_{k}(x) w_{n-k-1}(x)-\frac{i}{2} e^{i \beta \phi(x)} \delta_{n, 1}, \\
z_{n}(x, y)=\frac{i m}{4} \int_{y}^{x}\left(w_{n+1}\left(x^{\prime}\right)-e^{-i \beta \phi\left(x^{\prime}\right)} w_{n-1}\left(x^{\prime}\right)\right) d x^{\prime}
\end{gathered}
$$


Now imposing periodic boundary conditions at $\pm L$ simplifies the form of $\operatorname{tr} T^{\prime}(L,-L, \lambda)$. As a result, the first set of integrals will be the coefficients of $\operatorname{tr} \exp Z(L,-L, \lambda)$. The unimodularity of $T^{\prime}(L,-L, \lambda)$ implies that $Z(L,-L, \lambda)$ be traceless up to $O\left(|\lambda|^{-\infty}\right)$ terms, and so the $z_{n}(L,-L)^{\text {'s }}$ are real and $Z(L,-L, \lambda)$ is proportional to $\sigma_{3}$. Thus,

$$
\operatorname{tr} T^{\prime}(L,-L, \lambda)=\frac{1}{2} \cos \left(\sum_{n=1}^{\infty} \frac{z_{n}(L,-L)}{\lambda^{n}}-\frac{m \lambda L}{2}\right)+O\left(|\lambda|^{-\infty}\right), \quad \lambda \rightarrow \pm i \infty .
$$

Rewriting the cosine in terms of exponentials and neglecting the one which is $O\left(|\lambda|^{-\infty}\right)$, the result is,

$$
\ln \left(\operatorname{tr} T^{\prime}(L,-L, \lambda)\right)=i\left[\frac{m \lambda L}{2}-\frac{z_{1}(L,-L)}{\lambda}-\frac{z_{2}(L,-L)}{\lambda^{2}}-O\left(|\lambda|^{-3}\right)\right], \quad \lambda \rightarrow+i \infty,
$$

and this series generates the set of charges $\left\{z_{n}(L,-L)\right\}$ defined by (27), (28). These charges are purely imaginary, time independent, in involution with each other, and are elements of $\mathcal{A}_{S G}$. To construct elements of $\mathcal{A}_{S G}^{\mathbb{R}}$ it suffices to multiply all the charges by $i$. Note that (22),(29) also hold for $\alpha=-\pi / 2$ or $\lambda \rightarrow-i \infty$. The resulting set of charges in $\mathcal{A}_{S G}$ are complex conjugate to those found above and lead to the same elements of $\mathcal{A}_{S G}^{\mathbb{R}}$ on multiplication by $-i$.

The second distinct set of integrals comes from the expansion of $\operatorname{tr} T^{\prime}(L,-L, \lambda)$ as $\lambda \rightarrow i 0 \pm$. To find this, use is made of the invariance of (9),(17) under the replacement $\pi(x, t) \rightarrow \pi(x, t), \phi(x, t) \rightarrow-\phi(x, t), e^{\alpha} \rightarrow-e^{-\alpha}$. As a result

$$
\hat{T}^{\prime}\left(x, y,-\frac{1}{\lambda}\right)=T^{\prime}(x, y, \lambda),
$$

where $\hat{T}^{\prime}(x, y, \lambda)$ denotes the transition matrix for $\hat{\pi}(x, t)=\pi(x, t), \hat{\phi}(x, t)=-\phi(x, t)$. So taking $\lambda \rightarrow i 0 \pm$ in (31) amounts to taking $\mu:=-1 / \lambda \rightarrow \pm i \infty$ in $\hat{T}^{\prime}(L,-L, \mu)$. Therefore the analysis given above for $\lambda \rightarrow \pm i \infty$ can be used again on the replacement $\lambda \rightarrow-1 / \lambda, \phi(x, t) \rightarrow-\phi(x, t)$. The resulting conserved charges are,

$$
\hat{z}_{n}(L,-L)=\frac{i m}{4} \int_{-L}^{L}\left(\hat{w}_{n+1}\left(x^{\prime}\right)-e^{i \beta \phi\left(x^{\prime}\right)} \hat{w}_{n-1}\left(x^{\prime}\right)\right) d x^{\prime},
$$

with the $\hat{w}_{n}$ 's given by (27) on replacing $\phi \rightarrow-\phi$. Repeating the same procedure as above for this second set shows the $\left\{\hat{z}_{n}(L,-L)\right\}$ to be elements of $\mathcal{A}_{S G}^{\mathbb{R}}$.

The linear combination $H(L,-L):=\frac{2 m}{\beta^{2}}\left(z_{1}(L,-L)+\hat{z}_{1}(L,-L)\right)$ is of the form,

$$
H(L,-L)=\int_{-L}^{L}\left(\frac{1}{2} \pi^{2}+\frac{1}{2}\left(\frac{\partial \phi}{\partial x}\right)^{2}+\frac{m^{2}}{\beta^{2}}(1-\cos \beta \phi)\right) d x,
$$

and is identified as the Hamiltonian of the model. The sine-Gordon equation results from (20). Note that all the $z_{n}(L,-L), \hat{z}_{n}(L,-L)$ are zero for $n$ even. Also note that if $\phi$ is an even function of $x$ then the Hamiltonian density is itself even. In general the densities $z_{n}(L,-L)+\hat{z}_{n}(L,-L), \quad n=1,3, .$. have this property, ("parity even"). Finally extending $L \rightarrow \infty$ and demanding that $\phi$ have Schwartz boundary conditions, gives the theory on the whole line.

\section{$2.2 \quad$ Local boundary conditions}

The analysis given below was first provided by Sklyanin 11. The idea is to modify the Hamiltonian approach of the last subsection by introducing a new 'algebra of observables' $\mathcal{B}$, the form of which is not specified. This is to be contrasted with the precise definition of the algebra $\mathcal{A}$ given previously. By analogy with the transition matrix $T(x, y, t, \alpha)$, a $d \times d$ matrix with elements in $\mathcal{B}$ is introduced and called $\mathcal{T}(x, y, t, \alpha)$. This matrix is required to satisfy the equation, cf.(14),

$$
\left\{\mathcal{T}^{(1)}(\alpha), \mathcal{T}^{(2)}(\gamma)\right\}=\left[r_{12}(\alpha-\gamma), \mathcal{T}^{(1)}(\alpha) \mathcal{T}^{(2)}(\gamma)\right]+\mathcal{T}^{(1)}(\alpha) r_{12}(\alpha+\gamma) \mathcal{T}^{(2)}(\gamma)-\mathcal{T}^{(2)}(\gamma) r_{12}(\alpha+\gamma) \mathcal{T}^{(1)}(\alpha)
$$


$\forall \alpha, \gamma$, and the $x, y, t$ dependence has been suppressed. The Poisson bracket is defined in (13) with the exception that the range of the integral is changed to $\left[x_{-}, x_{+}\right]$, and it is understood that $x_{-} \leq y \leq x \leq x_{+}$. Antisymmetry of this bracket implies,

$$
\begin{array}{r}
r_{12}(\alpha)-r_{21}(\alpha)=0 \\
r_{12}(-\alpha)+r_{12}(\alpha)=0 .
\end{array}
$$

Constant representations of the Poisson bracket algebra $\mathcal{B}$ must also satisfy (34), hence

$$
\left[r_{12}(\alpha-\gamma), K_{ \pm}^{(1)}(\alpha) K_{ \pm}^{(2)}(\gamma)\right]+K_{ \pm}^{(1)}(\alpha) r_{12}(\alpha+\gamma) K_{ \pm}^{(2)}(\gamma)-K_{ \pm}^{(2)}(\gamma) r_{12}(\alpha+\gamma) K_{ \pm}^{(1)}(\alpha)=0,
$$

where $K_{ \pm}(\alpha)$ are $d \times d \mathbb{C}$ numerical matrices of the complex spectral parameter $\alpha$ and possibly some complex constants. From such a $K_{-}(\alpha)$, a $\mathcal{T}(x, y, t, \alpha)$ satisfying (34) can be built according to,

$$
\mathcal{T}(x, y, t, \alpha) \equiv T(x, y, t, \alpha) K_{-}(\alpha) T^{-1}(x, y, t,-\alpha),
$$

where $T(x, y, t, \alpha)$ is given by (ㄱ).

Multipying $\mathcal{T}(x, y, t, \alpha)$ on the left by $K_{+}(\alpha)$, evaluating the bracket of this with itself using (34), taking the trace and using the chain rule for functional differentiation gives,

$$
\left\{\ln \left(\operatorname{tr} K_{+}(\alpha) \mathcal{T}(x, y, t, \alpha)\right), \ln \left(\operatorname{tr} K_{+}(\gamma) \mathcal{T}(x, y, t, \gamma)\right)\right\}=0, \quad \forall \alpha, \gamma \in \mathbb{C}, \quad x, y \text { s.t } x_{-} \leq y \leq x \leq x_{+},
$$

i.e. an infinite set of involutive quantities. The question remains, however, as to whether these can be used to construct elements of the unspecified algebra $\mathcal{B}$. As was the case for the algebra $\mathcal{A}$, the requirement of smooth functional derivatives of elements of $\mathcal{B}$ imposes $y=x_{-}, x=x_{+}$. In principle, specification of the boundary conditions at $x_{ \pm}$on elements of phase space should be sufficient to determine $\mathcal{B}$ uniquely. In contrast to the periodic case, however, local boundary conditions of the form $\partial_{x} \Phi+\left.\partial_{\Phi} V(\Phi)\right|_{x=x_{ \pm}}=0$ do not impose any general form for functional derivatives of elements of $\mathcal{B}$. So, given boundary conditions at $x_{ \pm}$, the entire form of $\mathcal{B}$ cannot be deduced on symmetry grounds, there are no constraints on the forms of the $K_{ \pm}(\alpha)$, and a construction analogous to the one leading from (19) to (20) cannot be performed.

The way around this problem is to appeal to the zero-curvature formulation. For periodic boundary conditions, (12) can be regarded as a consistency check on the form of $\operatorname{tr} T(x, y, t, \alpha)$ such that it be an element of $\mathcal{A}$. In this case, such a check just imposes $y=-L, x=L$ which gives no new information on the structure of the algebra. If the same point of view is taken for the nonperiodic case, however, $K_{ \pm}(\alpha)$ become constrained by possible forms of boundary condition at $x_{ \pm}$. To see this, consider, $\partial_{t} K_{+}(\alpha) \mathcal{T}\left(x_{+}, x_{-}, t, \alpha\right)$ using (5), (37). The trace of this quantity should be zero if the Hamiltonian and zero-curvature approaches are to be consistent. This requirement leads to,

$$
K_{ \pm}(\alpha) V\left(x_{ \pm}, t, \pm \alpha\right)=V\left(x_{ \pm}, t, \mp \alpha\right) K_{ \pm}(\alpha), \quad \forall \alpha \in \mathbb{C}
$$

which contains nontrivial information as to the forms of the $K_{ \pm}(\alpha)$, for particular boundary conditions of the theory.

\section{Application to sine-Gordon theory}

In this section the formalism developed in subsection 2.2 is applied to the sine-Gordon equation. For sineGordon $d=2$ and the matrices $U, V$, appearing in the zero-curvature condition are given by (9), (10). The basis of End $\mathbb{C}^{2}$ is chosen to be the Pauli matrices and the two dimensional identity $\left(:=\sigma_{0}\right)$, and $K_{ \pm}(\alpha)$ is written in this basis as $D_{ \pm}^{\mu}(\alpha) \sigma_{\mu}, \mu=0, . ., 4$. It should be recalled that the standard Pauli matrices satisfy $\sigma_{i} \sigma_{j}=i \epsilon_{i j k} \sigma_{k}+\delta_{i j} \sigma_{0}, i, j, k=1, . ., 3$. Substituting $K_{ \pm}, V$ into (39) gives,

$$
\left.D_{ \pm}^{\mu}(\alpha)\left(\frac{\beta}{4 i} \frac{\partial \phi}{\partial x}\left[\sigma_{\mu}, \sigma_{3}\right]+\frac{m}{2 i} \sinh \alpha \sin \left(\frac{\beta \phi}{2}\right)\left\{\sigma_{\mu}, \sigma_{1}\right\}+\frac{m}{2 i} \cosh \alpha \cos \left(\frac{\beta \phi}{2}\right)\left[\sigma_{\mu}, \sigma_{2}\right]\right)\right|_{x=x_{ \pm}}=0 .
$$

The anticommutator implies $D_{ \pm}^{1}(\alpha)=0$, and so the most general form this equation can take is,

$$
\frac{\partial \phi}{\partial x}-\frac{2 m P_{ \pm}(\alpha)}{\beta} \sin \left(\frac{\beta \phi}{2}\right)-\left.\frac{2 m Q_{ \pm}(\alpha)}{\beta} \cos \left(\frac{\beta \phi}{2}\right)\right|_{x=x_{ \pm}}=0
$$


where,

$$
P_{ \pm}(\alpha)=\frac{i D_{ \pm}^{0}(\alpha)}{D_{ \pm}^{2}(\alpha)} \sinh \alpha, \quad Q_{ \pm}(\alpha)=\frac{D_{ \pm}^{3}(\alpha)}{D_{ \pm}^{2}(\alpha)} \cosh \alpha .
$$

Alternatively, $K_{ \pm}(\alpha)=D_{ \pm}^{0} \sigma_{0}$ implies,

$$
\left.\frac{R_{ \pm}(\alpha) m}{2 \beta} \sin \left(\frac{\beta \phi}{2}\right)\right|_{x=x_{ \pm}}=0
$$

with,

$$
R_{ \pm}(\alpha)=\frac{D_{ \pm}^{0}(\alpha) \sinh \alpha}{i} .
$$

From now on only the boundary at $x_{+}$will be considered; however similar arguments would apply to the other boundary. The subscripts \pm can therefore be suppressed. Eq.(41) would be in the form of a boundary condition that could be imposed on $\phi$ if $P(\alpha), Q(\alpha)$, were real and independent of $\alpha$. Similarly (43) would be a boundary condition if $R(\alpha)$ were independent of $\alpha$. Denoting these constants by $P, Q, R$ respectively, the form of $K(\alpha)$ is constrained even more. The result is,

$$
K(\alpha)=\frac{D^{2}(\alpha)}{i \sinh \alpha}\left(P \sigma_{0}+i \sinh \alpha \sigma_{2}+i Q \tanh \alpha \sigma_{3}\right),
$$

or,

$$
K(\alpha)=\frac{i R}{\sinh \alpha} \sigma_{0} .
$$

It can be verified that these satisfy $(36)$ so that $(38)$ holds with $(45),(46)$.

Since the $K(\alpha)$ are only defined up to an overall scale, it will be convenient to work with,

$$
\tilde{K}(\alpha):=\frac{P}{\sinh \alpha} \sigma_{0}+i \sigma_{2}+\frac{i Q}{\cosh \alpha} \sigma_{3},
$$

and

$$
\tilde{K}(\alpha):=\sigma_{0} .
$$

The normalization is fixed by requiring $\operatorname{det} \tilde{K}(\alpha) \rightarrow 1$ as $\operatorname{Re} \alpha \rightarrow \pm \infty$. So, by demanding that $\phi$ satisfy boundary conditions of the form,

$$
\frac{\beta}{2 m} \frac{\partial \phi}{\partial x}-P_{ \pm} \sin \left(\frac{\beta \phi}{2}\right)-\left.Q_{ \pm} \cos \left(\frac{\beta \phi}{2}\right)\right|_{x=x_{ \pm}}=0
$$

or,

$$
\phi-\left.\frac{2 n \pi}{\beta}\right|_{x=x_{ \pm}}=0, \quad n=1,2, \ldots,
$$

the associated $\tilde{K}_{ \pm}(\alpha)$ can be found and an infinite number of integrals in involution can be constructed. These will be the coefficients in the expansion of,

$$
\ln \left(\operatorname{tr} \tilde{K}_{+}(\ln (\lambda)) T^{\prime}\left(x_{+}, x_{-}, \lambda\right) \tilde{K}_{-}(\ln (\lambda)) T^{\prime-1}\left(x_{+}, x_{-}, 1 / \lambda\right)\right),
$$

as $\lambda \rightarrow \pm i \infty, i 0 \pm$. From these charges, elements of the algebra of observables $\mathcal{B}_{S G}^{\mathbb{R}}$ will be constructed as they were for periodic boundary conditions.

Now, consider the boundary value problem (2). Taking $x_{-}=-\infty$, (50) becomes the required Schwartz boundary condition. With $x_{+}=0$, (49) has the form of (2),(3) after relabelling constants, and the desired boundary value problem has been fitted into the developed formalism. Hence the conclusion that (2), (3) is integrable. It remains to construct the algebra of observables and identify a Hamiltonian for the problem from the infinity of integrals. This is done below. 
First put the problem (22),(3) on the finite interval $[-L, 0]$ and define

$$
\begin{aligned}
& \tilde{K}_{0}(\alpha):=\frac{P}{\sinh \alpha} \sigma_{0}+i \sigma_{2}+\frac{i Q}{\cosh \alpha} \sigma_{3}, \\
& \tilde{K}_{-L}(\alpha):=\sigma_{0}
\end{aligned}
$$

so that (51) becomes,

$$
\ln \left(\operatorname{tr} \tilde{K}_{0}(\ln (\lambda)) T^{\prime}(0,-L, \lambda) \tilde{K}_{-L} T^{\prime}(-L, 0,1 / \lambda)\right) .
$$

To expand this in an asymptotic series as $\lambda \rightarrow \pm i \infty$, use is made of (31),(21),(22) giving,

$$
\begin{gathered}
\ln \left(\operatorname{tr} \tilde{K}_{0}(\ln (\lambda)) \Omega(0)(I+W(0, \lambda)) \exp Z(0,-L, \lambda)(I+W(-L, \lambda))^{-1} \Omega^{-1}(-L) \hat{\Omega}(-L)\right. \\
\left.\times(I+\hat{W}(-L,-\lambda)) \exp \hat{Z}(-L, 0,-\lambda)(I+\hat{W}(0,-\lambda))^{-1} \hat{\Omega}^{-1}(0)+O\left(|\lambda|^{-\infty}\right)\right),
\end{gathered}
$$

where, $W, Z$ are defined by (23)-(28) and the hats indicate the same quantities but with $\phi \rightarrow-\phi$. Imposing the Schwartz boundary condition at $-L$ simplifies this further, the result being,

$$
\begin{aligned}
& \ln \left(\operatorname{tr} \tilde{K}_{0}(\ln (\lambda)) T^{\prime}(0,-L, \lambda) \tilde{K}_{-L}(\ln (\lambda)) T^{\prime-1}(0,-L, 1 / \lambda)\right) \\
& \quad=\ln \left(\operatorname{tr}(I+\hat{W}(0,-\lambda))^{-1} \Omega(0) \tilde{K}_{0}(\ln (\lambda)) \Omega(0)(I+W(0, \lambda)) \exp (Z(0,-L, \lambda)-\hat{Z}(0,-L,-\lambda))\right)
\end{aligned}
$$

$\bmod \left(O|\lambda|^{-\infty}\right)$ as $\lambda \rightarrow \pm i \infty$. From (24), it follows that,

$$
Z(0,-L, \lambda)-\hat{Z}(0,-L,-\lambda)=i \sum_{n=-1}^{\infty} \frac{a_{n}(0,-L)}{\lambda^{n}} \sigma_{3}-\sum_{n=1}^{\infty} \frac{b_{n}(0,-L)}{\lambda^{n}} I,
$$

where $z_{n}(0,-L)-(-1)^{n} \hat{z}_{n}(0,-L)=a_{n}(0,-L)+i b_{n}(0,-L), n=1,2, . .$, and $a_{0}(0,-L)=0$, $a_{-1}(0,-L)=-m \lambda L / 2$. Hence, only the diagonal terms in $(I+\hat{W}(0,-\lambda))^{-1} \Omega(0) \tilde{K}_{0}(\ln (\lambda)) \Omega(0)(I+W(0, \lambda))$ will contribute to the trace. Defining,

$$
\operatorname{diag}\left((I+\hat{W}(0,-\lambda))^{-1} \Omega(0) \tilde{K}_{0}(\ln (\lambda)) \Omega(0)(I+W(0, \lambda))=\sum_{n=0}^{\infty}\left(\frac{i c_{n}(0)}{\lambda^{n}} \sigma_{3}+\frac{d_{n}(0)}{\lambda^{n}} I\right),\right.
$$

where $(52),(56)$ and the involutions,

$$
\begin{aligned}
\overline{(I+\hat{W}(0,-\bar{\lambda}))^{-1} \Omega(0) \tilde{K}_{0}(\ln (\bar{\lambda})) \Omega(0)(I+W(0, \bar{\lambda}))} & \\
& =\sigma_{2}(I+\hat{W}(0,-\lambda))^{-1} \Omega(0) \tilde{K}_{0}(\ln (\lambda)) \Omega(0)(I+W(0, \lambda)) \sigma_{2}, \\
\frac{\exp (Z(0,-L, \bar{\lambda}))-\hat{Z}(0,-L,-\bar{\lambda})}{} & =\sigma_{2} \exp (Z(0,-L, \lambda)-\hat{Z}(0,-L,-\lambda)) \sigma_{2},
\end{aligned}
$$

are used to show that the $\left\{c_{n}(0), d_{n}(0)\right\}$ are real. Thus (56) has the form,

$$
\ln \left(2 \sum_{n=0}^{\infty} \frac{d_{n}(0)}{\lambda^{n}} \cos \left(\sum_{m=-1}^{\infty} \frac{a_{m}(0,-L)}{\lambda^{m}}\right)-2 \sum_{n=0}^{\infty} \frac{c_{n}(0)}{\lambda^{n}} \sin \left(\sum_{m=-1}^{\infty} \frac{a_{m}(0,-L)}{\lambda^{m}}\right)\right)-\sum_{k=1}^{\infty} \frac{b_{k}(0,-L)}{\lambda^{k}}
$$

$\bmod \left(O\left(|\lambda|^{-\infty}\right)\right.$ as $\lambda \rightarrow \pm i \infty$, the Laurent expansion of which has real coefficients. Now consider the expansion of this as $\lambda \rightarrow+i \infty$. Rewriting the sin, cos in terms of exponentials and discarding the ones which are $O\left(|\lambda|^{-\infty}\right)$, 61) becomes,

$$
i \sum_{m=-1}^{\infty} \frac{a_{m}(0,-L)}{\lambda^{m}}-\sum_{k=1}^{\infty} \frac{b_{k}(0,-L)}{\lambda^{k}}+\ln \left(\sum_{n=0}^{\infty} \frac{d_{n}(0)+i c_{n}(0)}{\lambda^{n}}\right),
$$

as $\lambda \rightarrow+i \infty$. From (58), (52), (23), (25) one finds $c_{0}=1, d_{0}=0$, so that the logarithm can be expanded as a power series. The coefficient of $(62)$ at $O(1 / \lambda)$ is $i\left(a_{1}-d_{1}\right)-\left(b_{1}-c_{1}\right)$. This is, in principle, a complex 
valued functional and is defined to be an element of $\mathcal{B}_{S G}$. Indeed, this will be the case to all orders. It remains to construct the integrals of motion which are elements of $\mathcal{B}_{S G}^{\mathbb{R}}$. To do this, use is made of the $\lambda \rightarrow-i \infty$ expansion of (61) which has coefficients at each order in $\lambda$ that are complex conjugate to those in the $\lambda \rightarrow+i \infty$ expansion. Hence, not only are the complex integrals in involution with one another, but also the complex conjugated ones too. (This is an obvious consequence of the real coordinates on $\mathcal{M}_{S G}$ ). As a result, the real and imaginary part of each integral is separately in involution with the real and imaginary parts of all the other integrals. Therefore once a Hamiltonian for the system is identified as being the real or imaginary part at some order in $\lambda$, then the real or imaginary parts of the others can be chosen at will. Thus, by multiplying terms by $i$ an infinite number of elements of $\mathcal{B}_{S G}^{\mathbb{R}}$ in involution, can be constructed. At $O(1 / \lambda)$ in (62), the first element of $\mathcal{B}_{S G}$ is,

$$
\frac{-i \beta^{2}}{2 m}\left(\int_{-L}^{0}\left[\frac{1}{2} \pi^{2}+\frac{1}{2}\left(\frac{\partial \phi}{\partial x}\right)^{2}+\frac{m^{2}}{\beta^{2}}(1-\cos \beta \phi)\right] d x+\frac{4 P m}{\beta^{2}} \cos \frac{\beta \phi(0)}{2}-\frac{4 Q m}{\beta^{2}} \sin \frac{\beta \phi(0)}{2}\right) .
$$

Multiplication of this by $2 m i / \beta^{2}$ defines an element of $\mathcal{B}_{S G}^{\mathbb{R}}$ and is identified as the Hamiltonian of the system (2), (3) defined over the interval $[-L, 0]$. By imposing Schwartz boundary conditions at $-L$ the integral can be extended to the whole half-axis.

The $O\left(1 / \lambda^{2}\right)$ term is found to be real and equal to $4\left(P^{2}+Q^{2}\right)$, i.e. a trivial integral of motion. The coefficient at $O\left(1 / \lambda^{3}\right)$ is purely imaginary and is made up of the integral over [ $\left.-L, 0\right]$ of the next "parity even" density plus a boundary term. It is not clear as to whether the series continues to alternate between real and imaginary terms. However, it will be the case that the integrals at $O\left(1 / \lambda^{2 n}\right), n=1,2, .$. , will depend purely on the boundary at $x=0$.

Finally it remains to return to (54) to calculate its expansion as $\lambda \rightarrow i 0 \pm$. Replacing $\lambda$ by $-1 / \mu$ in (54) and using (31) one finds that the analysis for $\lambda \rightarrow \pm i \infty$ will hold on replacing $\phi \rightarrow-\phi, Q \rightarrow-Q$. So all the integrals previously found will form another infinite set on imposition of this transformation. However the first three are invariant under this, and hence it is likely to be the case in general.

\section{Conclusions}

The system (2),(3) conjectured to be integrable by Ghoshal and Zamolodchikov has been fitted in to a formalism developed by Sklyanin for integrable equations with local boundary conditions. It has been shown that an infinite number of integrals of motion, in involution, do exist. These charges fall into two classes: those consisting of integrals of "even parity" densities plus a boundary term, and those depending purely on the field and its derivatives at the boundary.

This analysis fits into the scheme developed by Khabibullin on symmetrical reductions of Bäcklund transformations, but it remains to continue the problem evenly to the whole axis with a "point-spin" at the origin along the lines of [13, 14]. The analysis of the solutions of such a system is an interesting open problem.

Acknowledgments: I would like to thank Peter Bowcock, Ed Corrigan, Patrick Dorey, and Martin Speight for useful discussions. I also acknowledge the financial support of the U.K. Engineering and Physical Sciences Research Council. 


\section{References}

[1] I.V. Cherednik, "Factorizing particles on a half line and root systems", Theor. Math. Phys. 61(1) (1984) 977-983.

[2] A. Fring and R. Köberle, "Factorized scattering in the presence of reflecting boundaries", Nucl. Phys. B421 (1994) 159.

[3] S. Ghoshal and A.B.Zamolodchikov, "Boundary state and boundary S matrix in two-dimensional integrable field theory", Int. J. Mod. Phys. A9 (1994) 3841.

[4] R. Sasaki, "Reflection bootstrap equations for Toda field theory", in Interface between physics and mathematics eds. W. Nahm, Jian-Min Shen, (World Scientific 1994), hep-th/9311027.

[5] E. Corrigan, P.E. Dorey, R.H. Rietdijk, R. Sasaki, "Affine Toda field theory on a half line", Phys. Lett. B333 (1994) 83, "Apects of affine Toda field theory on a half line", DTP 94/29 hep-th/9407148.

[6] S. Ghoshal, "Boundary S matrix of the O(N) nonlinear sigma model", Phys. Lett. B334 (1994) 363.

[7] V.E. Korepin, N.M. Bogoliubov, A.G. Izergin, Quantum inverse scattering method and correlation functions (C.U.P. Cambridge, U.K. 1992).

[8] E.K. Sklyanin, "Boundary conditions for integrable quantum systems", J. Phys. A21 (1988) 2375-2389.

[9] L. Mezincescu and R. Nepomechie, "Fusion procedure for open chains", J. Phys. A25(1992) 2533-2543.

[10] H.J. de Vega and A. González Ruiz, "Boundary K-matrices for the XYZ, XXZ and XXX spin chains, LPTHE-PAR 93-29 hep-th/9306089.

[11] E.K. Sklyanin, "Boundary conditions for integrable equations", Funct. Anal. Appl. 21 (1987) 164.

[12] L.D. Faddeev and L.A. Takhtajan, Hamiltonian methods in the theory of solitons (Springer-Verlag, Berlin, Germany, 1987).

[13] P.N. Bibikov and V.O. Tarasov, "Boundary value problem for the nonlinear Schrödinger equation", Theor. Math. Phys. 79(3) (1989) 570-579.

[14] V.O. Tarasov, "The integrable initial-boundary value problem on a semiline: nonlinear Schrödinger and sine-Gordon equations", Inverse Problems 7 (1991) 435-449.

[15] I.T. Khabibullin, "Integrable initial-boundary value problems", Theor. Math. Phys. 86(1) (1991) 28-36.

[16] I.T. Khabibullin, "The Backlund transformation and integrable initial-boundary value problems", Math. Notes 49(3-4) (1991) 418-423.

[17] R.F. Bikbaev and A.R. Its, "Algebrogeometric solutions of a boundary value problem for the nonlinear Schrödinger equation", Math. Notes 45(5-6) (1989) 349-354.

[18] R.F. Bikbaev and A.R. Its, "Algebrogeometric solutions of the nonlinear boundary value problem on a segment for the sine-Gordon equation", Math. Notes 52(3-4) (1992) 1005-1011.

[19] H. Saleur, S. Skorik, N.P. Warner, "The boundary sine-Gordon theory: classical and semiclassical analysis", USC-94-013 hep-th/9408004.

[20] V.I. Arnold, Mathematical methods of classical mechanics (Springer-Verlag, New York, 1978). 